\title{
ANÁLISE COMPARATIVA DO NÚMERO DE ÓBITOS POR SUICÍDIO ENTRE A CIDADE DE ARAGUAÍNA E O ESTADO DO TOCANTINS NO PERÍODO DE 2015 A 2020
}

\author{
COMPARATIVE ANALYSIS OF THE NUMBER OF DEATHS BY \\ SUICIDE BETWEEN THE CITY OF ARAGUAÍNA AND TOCANTINS \\ STATE IN THE PERIOD FROM 2015 TO 2020
}

Maria Laryssa Ferreira da Silva ${ }^{1}$ Mariana Coutinho Martins Ferreira ${ }^{2}$ Jane da Silva Propércio ${ }^{3}$

\begin{abstract}
RESUMO: Objetivo: O suicídio é um fenômeno que ocorre em todo o mundo. Estima-se que anualmente mais de 800 mil pessoas cometem suicídio no mundo, o que equivale a um suicídio a cada 40 segundos (WHO, 2019). Esse trabalho tem como objetivo analisar o perfil epidemiológico dosóbitos por suicídio no município de Araguaína de forma comparativa com o Estado do Tocantins, relacionando a prevalência do número de casos com o ano de notificação, sexo, faixa etária, raça, local de ocorrência e o tipo de lesão autoprovocada que causou o óbito. Método: Trata-se de um estudo observacional, descritivo e retrospectivo dos casos de óbitos por lesões autoprovocadas voluntariamente no município de Araguaína, de forma comparativa com o Estado do Tocantins, no período de 2015 a 2020, por meio dos dados fornecidos pelo Sistema de Informação em Mortalidade (SIM), publicados pelo DataSUS e disponíveis na página online do Ministério da Saúde. Resultados: Os resultados demostram 640 casos de óbitos por suicídio no Estado do Tocantins durante o período estudado, e no município de Araguaína 66 casos, representando apenas $10,31 \%$ do total de casos do Estado do Tocantins. O método de lesão autoprovocada mais utilizado foi o enforcamento, com predomínio do sexo masculino. Conclusão: Diante deste contexto, e por ser um grande impasse na saúde pública, é imprescindível o desenvolvimento de políticas públicas e estratégias multidisciplinares, além de conscientização da população e capacitação dos profissionais da saúde para ajudarem na identificação dos grupos de risco.
\end{abstract}

\footnotetext{
${ }^{1}$ Acadêmica do Curso de Medicina do Centro Universitário Tocantinense Presidente Antônio Carlos UNITPAC. Araguaína - Tocantins, Brasil. E-mail: E-mail: marialaryssa292@gmail.com.

${ }^{2}$ Acadêmica do Curso de Medicina do Centro Universitário Tocantinense Presidente Antônio Carlos UNITPAC. Araguaína - Tocantins, Brasil. E-mail: marianacoutinhomf@hotmail.com.

${ }_{3}$ Médica docente do Centro Universitário Tocantinense Presidente Antônio Carlos - UNITPAC, especialista emClínica Geral. Araguaína - Tocantins, Brasil. E-mail: jpropercio@yahoo.com.br.
} 
Palavras chave: Epidemiologia. Mortalidade. Saúde mental.

ABSTRACT: Objective: Suicide is a worldwide phenomenon. It is estimated that more than 800 thousand people commit suicide in the world annually, which is equivalent to one suicide every 40 seconds (WHO, 2019). This study aims to analyze the epidemiological profile of deaths by suicide in the city of Araguaina compared to Tocantins State, relating to the prevalence of the number of cases with year of notification, sex, age group, race, place of occurrence and the type of self-harm that caused the death. Method: This is an observational, descriptive and retrospective study of death cases from self-inflicted injuries in the city of Araguaína, in a comparative to Tocantins States, in the period from 2015 to 2020, through the data provided by the Information System in Mortality (SIM), published by DataSUS, available on the Ministry of Health website. Results: The results show 640 cases of deaths by suicide in the State of Tocantins during these years, besides, in Araguaina city there were only 66 cases, representing only $10.31 \%$ of the total cases in the State of Tocantins. The most used method of self-harm was hanging, with a male predominance. Conclusion: In this context and because it is a majorimpasse in public health, it is essential to develop public policies and multidisciplinary strategies, in addition to raising awareness among the population and training health professionals to help identify groups of risk.

Keywords: Epidemiology. Mortality. Mental health. 


\section{INTRODUÇÃO}

O suicídio é um grave problema de saúde pública. A cada 40 segundos uma pessoa morre por suicídio no mundo (WHO, 2019). Tal ação pode ser compreendida como o resultado de uma convergência de fatores de risco genéticos, psicológicos, sociais e culturais, podendo estar associado a experiências de traumas e perdas, bem como a grupos vulneráveis que sofrem discriminação: indígenas, comunidade LGBTI+, imigrantes e refugiados (OMS, 2020).

O principal fator de risco para a ocorrência do comportamento suicida é a préexistência de transtornos mentais (WHO, 2014). Entre os transtornos mais comuns estão a depressão, o transtorno de humor bipolar e a dependência de álcool e de outras drogas psicoativas. Outros transtornos como a esquizofrenia também representam um importante fator de risco. E quandomais de uma dessas condições combinam-se, as chances do indivíduo cometer suicídio aumentam consideravelmente (BERTOLOTE et al., 2010).

Inúmeros métodos podem ser utilizados para a prática do autoextermínio, são eles: ingestão de medicamentos, produtos químicos nocivos e agrotóxicos; inalação de gases e vapores tóxicos; enforcamento; afogamento; uso de arma de fogo ou arma branca; dispositivos explosivos; exposição a chamas ou fogo; precipitação de locais elevados e objetos em movimento; entre outros (CID-10, 2019).

Desde a criação da Portaria $n^{\circ} 1.876$ em 2006, o Brasil conta com diretrizes para a prevenção do suicídio, estabelecendo que as medidas devem ser realizadas em todas as unidadesda federação e de uma forma multidisciplinar, incluindo desde a promoção de qualidade de vidaa medidas de recuperação da saúde (BRASIL, 2019). No entanto, há até hoje várias objeções, desde governamentais a sociais, para consolidar de forma eficiente a prevenção do suicídio.

Estudos realizados mostram que a temática ainda é pouco abordada em algumas regiões do Brasil, em especial na região Norte (LOVISI et al., 2009; FERREIRA et al., 2014). O conhecimento da distribuição geográfica e temporal dos óbitos por suicídios, em cada UnidadeFederativa e município do Brasil, é de grande 
importância para a compreensão das vulnerabilidades, e então implementação de ações e medidas de controle.

Perante a falta de estudos sobre o tema e informações acerca dos óbitos por suicídio no Estado do Tocantins, o objetivo desse trabalho foi analisar o perfil epidemiológico dos óbitos por suicídio no município de Araguaína de forma comparativa com o Estado do Tocantins no período de 2015 a 2020. E, dessa forma, relacionar a prevalência dos óbitos por suicídio com as variáveis: ano de notificação, sexo, faixa etária, raça, local de ocorrência e o tipo de lesão autoprovocada que causou o óbito.

\section{METODOLOGIA}

Trata-se de um estudo observacional, descritivo e retrospectivo dos casos de óbitos por lesões autoprovocadas, voluntariamente, no município de Araguaína, de forma comparativa com o Estado do Tocantins, no período de 2015 a 2020.

A pesquisa foi desenvolvida através dos dados fornecidos pelo Sistema de Informação em Mortalidade (SIM), publicados pelo DataSUS e disponíveis na página online do Ministério da Saúde. Foram utilizados todos os casos de notificação de óbitos por lesões autoprovocadas intencionalmente, segundo os códigos X60-X84, conforme a décima revisão da Classificação Internacional de Doenças (CID-10).

Variáveis epidemiológicas estudadas: o número de suicídio por ano, sexo, faixa etária, raça, local de ocorrência e o tipo de lesão autoprovocada que causou o óbito. Os dados foram coletados e transferidos para planilhas do Microsoft Office Excel 2013, que permitiu a análise estatística da pesquisa. Para a confecção das tabelas, foi utilizado o Microsoft Word 2013 e também a literatura de base sobre o tema para subsidiar a discussão dos dados. 


\section{RESULTADOS}

Entre os anos de 2015 a 2020 foram notificadas 640 mortes por suicídio no Estado do Tocantins, e no município de Araguaína foram notificados 66 casos, correspondendo a 10,31\%dos casos de todo o Estado.

Durante esse período, o ano de 2020 teve a menor taxa de mortalidade por suicídio. NoEstado do Tocantins, houve uma queda de $50 \%$ dos casos e, na cidade de Araguaína, houve 1 caso a menos em relação ao ano anterior (Tabela 1).

Tabela 1 - Casos de óbitos por suicídio segundo o ano de notificação no Estado do Tocantinse na cidade de Araguaína.

\begin{tabular}{ccccc}
\hline \multirow{2}{*}{ Ano de notificação } & \multicolumn{2}{c}{ Tocantins } & \multicolumn{2}{c}{ Araguaína } \\
\cline { 2 - 5 } & $\mathbf{N}$ & $\%$ & $\mathbf{N}$ & $\%$ \\
\hline 2015 & 99 & 15,47 & 11 & 16,67 \\
2016 & 101 & 15,78 & 11 & 16,67 \\
2017 & 115 & 17,97 & 14 & 21,21 \\
2018 & 124 & 19,38 & 11 & 16,67 \\
2019 & 134 & 20,94 & 10 & 15,15 \\
2020 & 67 & 10,47 & 9 & 13,64 \\
Total & 640 & 100,0 & 66 & 100,0 \\
\hline
\end{tabular}

Fonte: SIM, 2021.

$\mathrm{Na}$ tabela 2 observa-se o número de óbitos por suicídio, no Estado do Tocantins, de acordo com o sexo, faixa etária, raça, local de ocorrência e tipo de lesão autoprovocada. Percebe-se que dos 640 casos notificados do total, 511 eram do sexo masculino (79,84\%), e apenas 129 eram do sexo feminino $(20,16 \%)$.

Quanto à faixa etária, há o predomínio de óbitos por lesões autoprovocadas em jovens de 20 a 29 anos, com 138 casos registrados (21,56\%), acompanhado de 132 casos entre indivíduos dos 30 a 39 anos $(20,63 \%)$, seguido das demais faixas etárias. Referente à raça, 428 indivíduos eram da raça parda $(66,88 \%), 121$ da raça branca (18,90\%), 53 da raça preta (8,28\%), 22 indígenas $(3,44 \%)$ e apenas 1 da raça amarela $(0,16 \%)$. Por outro lado, 15 indivíduos não tiveram suas raças notificadas $(2,34 \%)$. 
Em relação ao local de ocorrência, o domicílio foi o local mais frequente com 424 óbitos $(66,25 \%)$, subsequente de outros locais não especificados com 125 óbitos $(19,53 \%)$. Os hospitais, as vias públicas e outros estabelecimentos de saúde obtiveram $59(9,22 \%), 28(4,38 \%)$ e $4(0,62 \%)$ casos de óbitos notificados, respectivamente.

Entre os tipos de lesão autoprovocada escolhidos pelos indivíduos houve predominância pelo enforcamento. Em todo o Estado foram 481 óbitos, o que corresponde a $75,16 \%$ das 640 mortes do total. O segundo método mais utilizado foi a arma de fogo com 66 notificações (10,31\%), acompanhada da intoxicação exógena com 57 (8,91\%) e outros métodos não especificados com 36 (5,62\%).

Tabela 2 - Casos notificados de óbitos por suicídio segundo sexo, faixa etária, raça, local deocorrência e tipo de lesão autoprovocada, Tocantins, 2015 a 2020.

\begin{tabular}{ccc}
\hline Sexo & $\mathbf{N}$ & $\%$ \\
\hline Feminino & 129 & 20,16 \\
Masculino & 511 & 79,84 \\
Ignorado & - & - \\
Total & 640 & 100,0 \\
\hline Faixa Etária & $\mathbf{N}$ & $\%$ \\
\hline $05-09$ & 1 & 0,16 \\
$10-14$ & 11 & 1,71 \\
$15-19$ & 71 & 11,09 \\
$20-29$ & 138 & 21,56 \\
$30-39$ & 132 & 20,63 \\
$40-49$ & 105 & 16,41 \\
$50-59$ & 83 & 12,97 \\
$60-69$ & 45 & 7,03 \\
$70-79$ & 38 & 5,94 \\
80 ou mais & 16 & 2,50 \\
Total & 640 & 100,0 \\
\hline Raça & $\mathbf{N}$ & $\%$ \\
\hline Branca & 121 & 18,90 \\
Preta & 53 & 8,28 \\
Amarela & 1 & 0,16 \\
Parda & 428 & 66,88 \\
Indígena & 22 & 3,44 \\
Ignorada & 15 & 2,34 \\
Total & 640 & 100,0 \\
\hline
\end{tabular}




\begin{tabular}{ccc}
\hline Local de ocorrência & $\mathbf{N}$ & $\%$ \\
\hline Hospital & 59 & 9,22 \\
Outros estabelecimentos & 4 & 0,62 \\
de saúde & & \\
Domicílio & 424 & 66,25 \\
Via pública & 28 & 4,38 \\
Outros & 125 & 19,53 \\
Aldeia indígena & - & - \\
Ignorada & - & - \\
Total & 640 & 100,0 \\
\hline Tipo de lesão & $\mathbf{N}$ & $\%$ \\
autoprovocada & & \\
\hline Intoxicação exógena & 57 & 8,91 \\
Enforcamento & 481 & 75,16 \\
Arma de fogo & 66 & 10,31 \\
Outros & 36 & 5,62 \\
Total & 640 & 100,0 \\
\hline
\end{tabular}

Fonte: SIM, 2021.

Outrossim, na tabela 3 identifica-se o número de suicídios quanto as variáveis: sexo, faixa etária, raça, local de ocorrência e tipo de lesão autoprovocada, no município de Araguaína. Analisou-se que dos 66 casos notificados do total, 53 eram do sexo masculino $(80,30 \%)$ e 13 eram do sexo feminino $(19,70 \%)$.

Concernente a variável faixa etária, o predomínio de suicídios foi dos 40 aos 49 anos, com 19 casos notificados (28,79\%), seguido de 14 casos em jovens dos 20 aos 29 anos $(21,21 \%)$ e 11 casos entre os 30 - 39 anos (16,67\%), acompanhado das outras idades. Sobre à raça, 42 indivíduos eram da raça parda (63,64\%), 20 da raça branca $(30,30 \%)$ e 2 da raça preta $(3,03 \%)$. As raças amarela e indígena não obtiveram nenhuma notificação, e 2 indivíduos não tiveram suas raças registradas $(3,03 \%)$.

Conforme o local de ocorrência, a cidade de Araguaína, assim como o Estado do Tocantins, apresentou em primeiro lugar o domicílio, com 47 suicídios notificados (71,21\%), seguido de outros locais não especificados com 8 notificações $(12,12 \%)$ e os hospitais com 7 (10,61\%). As vias públicas e os outros estabelecimentos de saúde obtiveram 2 casos cada (3,03\%), o que equivale a $6,06 \%$ dos 66 casos do total. 
E em relação aos tipos de lesão autoprovocada a predominância se mantêm no enforcamento, com 49 mortes registradas $(74,24 \%)$. O segundo método mais utilizado foi a arma de fogo com 8 casos $(12,12 \%)$, acompanhada da intoxicação exógena com 7 casos $(10,60 \%)$, além de 2 notificações por outros métodos não especificados $(3,04 \%)$, totalizando assim, os 66 casos de óbitos por suicídio em todo o município.

Tabela 3 - Casos notificados de óbitos por suicídio segundo sexo, faixa etária, raça, local deocorrência e tipo de lesão autoprovocada, Araguaína - TO, 2015 a 2020.

\begin{tabular}{ccc}
\hline Sexo & $\mathbf{N}$ & $\%$ \\
\hline Feminino & 13 & 19,70 \\
Masculino & 53 & 80,30 \\
Ignorado & - & - \\
Total & 66 & 100,0 \\
\hline Faixa Etária & $\mathbf{N}$ & $\%$ \\
\hline $05-09$ & - & - \\
$10-14$ & 1 & 1,52 \\
$15-19$ & 9 & 13,64 \\
$20-29$ & 14 & 21,21 \\
$30-39$ & 11 & 16,67 \\
$40-49$ & 19 & 28,79 \\
$50-59$ & 5 & 7,58 \\
$60-69$ & 2 & 3,02 \\
$70-79$ & 3 & 4,55 \\
80 ou mais & 2 & 3,02 \\
Total & 66 & 100,0 \\
\hline Raça & $\mathbf{N}$ & $\%$ \\
\hline Branca & 20 & 30,30 \\
Preta & 2 & 3,03 \\
Amarela & - & - \\
Parda & 42 & 63,64 \\
Indígena & - & - \\
Ignorada & 2 & 3,03 \\
Total & 66 & 100,0 \\
\hline Local de ocorrência & $\mathbf{N}$ & $\%$ \\
\hline Hospital & 7 & 10,61 \\
Outros estabelecimentos & 2 & 3,03 \\
de saúde & & 71,21 \\
Domicílio & 2 & 3,03 \\
Via pública & 8 & 12,12 \\
Outros & &
\end{tabular}




\begin{tabular}{ccc}
$\begin{array}{c}\text { Aldeia indígena } \\
\text { Ignorada } \\
\text { Total }\end{array}$ & - & - \\
\hline Tipo de lesão & 66 & - \\
autoprovocada & $\mathbf{N}$ & 100,0 \\
\hline Intoxicação exógena & 7 & $\%$ \\
Enforcamento & 49 & 10,60 \\
Arma de fogo & 8 & 74,24 \\
Outros & 2 & 12,12 \\
Total & 66 & 3,04 \\
& & 100,0 \\
\hline
\end{tabular}

Fonte: SIM, 2021.

\section{DISCUSSÃO}

A análise realizada, comparando os casos de suicídio no município de Araguaína com os do Estado do Tocantins nos anos de 2015 a 2020, evidenciou que durante esse período, 2017 e 2019 foram os anos com maiores índices de mortes por suicídio, em Araguaína com 14 casos e no Tocantins com 134 casos, respectivamente. Em contrapartida, 2020 foi o ano com menor número de suicídios, tanto no Estado do Tocantins com 67 notificações, quanto no município de Araguaína com 09 notificações.

Embora no ano de 2020 a pandemia de COVID-19 tenha tornado as pessoas mais vulneráveis a problemas de saúde mental como depressão e ansiedade e aumentado os riscos de comportamento suicida (GREFF et al., 2020), os casos de suicídio diminuíram significativamente, principalmente no Estado do Tocantins, quando comparado a Araguaína. Essa redução pode ser justificada pelo fortalecimento dos laços socioafetivos durante a pandemia, o chamado "pullingtogether effect", no qual durante o compartilhamento de determinadas experiências, as pessoas apoiam-se, aumentando a conexão entre elas (REGER et al., 2020). Ademais, o maior convívio familiar e o aumento nas buscas por atendimento com psiquiatras e psicólogos (ABP, 2020), atuaram como fatores protetivos e corroboraram essa diminuição de casos. 
Em relação ao gênero, observou-se um predomínio nos óbitos por suicídio no sexo masculino, cerca de $79,84 \%$ dos casos no Estado do Tocantins e aproximadamente $80,30 \%$ dos casos no município de Araguaína. A principal teoria usada para justificar essa prevalência é deque os homens têm mais acesso a objetos letais, como armas de fogo, e por isso maior êxito nastentativas. Além de serem mais sensíveis a fatores estressantes, como crises financeiras. Enquanto as mulheres, apesar de realizarem mais tentativas de suicídio, escolhem meios mais facilmente reversíveis e menos invasivos - como a intoxicação exógena - e também buscam mais apoio em situações de crise (CALIXTO FILHO et al., 2016).

No que tange a faixa etária, foi a única divergência entre o Estado e o município pois noEstado do Tocantins a prevalência é maior entre os 20 - 29 anos de idade, cerca de $21,56 \%$ dos casos, enquanto no município de Araguaína a faixa etária mais prevalente é entre os 40 - 49 anos de idade, aproximadamente $28,79 \%$ dos casos. Os índices elevados entre a população economicamente ativa podem ser explicados por frustrações no mercado de trabalho como desemprego, além do maior consumo de drogas, aumentando o risco de suicídio (SCHLÖSSER et al 2014).

Quanto à raça, no Tocantins e em Araguaína é prevalente a raça parda, com $66,88 \%$ e $63,64 \%$ dos casos, respectivamente. Esse fato pode ser justificado pela miscigenação característica do país (PNAD, 2019).

Em referência ao local de ocorrência, o domicílio foi o lugar mais frequente em $66,25 \%$ das notificações no Estado do Tocantins e do mesmo modo ocorreu na cidade de Araguaína com $71,21 \%$ dos casos notificados. Pode ser devido ao maior acesso aos meios de consumaçãodesse ato nesse lugar e de ser considerado o local mais propício para a realização (RIBEIRO et al., 2018).

Concernente ao tipo de lesão autoprovocada pelos indivíduos nos suicídios, o enforcamento foi o que teve maior prevalência, com $75,16 \%$ dos casos no Estado do Tocantins e $74,24 \%$ do total no município de Araguaína. Seguido pelo uso de armas de fogo e intoxicação exógena, evidenciando assim, a facilidade no acesso a esses métodos e as irregularidades no controle e fiscalização desses (CAMPOS et al., 2019).

Considerando que o suicídio é um grave problema de saúde pública, é essencial conhecer as principais situações de risco para a criação de medidas de 
prevenção efetivas, que podem abranger desde o impedimento do fácil acesso à comercialização de armas de fogo e pesticidas, ao correto preenchimento das fichas de notificação compulsória dos casos de tentativas de suicídio e a capacitação dos profissionais da saúde para torná-los aptos a reconhecerem indivíduos com comportamento suicida, corroborando assim para que o êxito na prevenção desse impasse seja alcançado (D'OLIVEIRA et al., 2006).

\section{CONCLUSÃO}

O presente estudo demostrou por meio da avaliação do perfil epidemiológico dos óbitos por suicídio, no estado no Tocantins, a supremacia de casos notificados em indivíduos do sexo masculino, da raça parda, dos 20 - 29 anos de idade, com predomínio do domicílio como o local de ocorrência e o enforcamento como o principal tipo de lesão autoprovocada que causou o óbito. Sendo o ano de 2019, com maior índice de mortes por esse ato. Em contrapartida, no município de Araguaína, 2017 foi o ano com maior número de casos notificados e a faixa etária predominante foi dos 40 - 49 anos de idade, sendo essas únicas variáveis divergentes entre eles.Diante deste contexto e por ser um grande impasse na saúde pública, é imprescindível desenvolver políticas públicas e estratégias multidisciplinares que forneçam aos indivíduos mais susceptíveis ao comportamento suicida, uma assistência integral, além de conscientizar a população e capacitar os profissionais da saúde para ajudarem na identificação dos grupos de risco, auxiliando assim, na redução dos índices de casos de suicídio tanto no município de Araguaína quanto no Estado do Tocantins. 


\section{REFERÊNCIAS BIBLIOGRÁFICAS}

ALMEIDA, Felipe Mateus de. O suicídio: Contribuições de Émile Durkheim e Karl Marx paraa compreensão desse fenômeno na contemporaneidade. Revista Aurora, v. 11, n. 1, p. 119138, 2018.

BERTOLOTE, José anoel; MELLO-SANTOS, Carolina de; BOTEGA, Neury José. Detecçãodo risco de suicídio nos serviços de emergência psiquiátrica. Brazilian Journal of Psychiatry,v. 32, p. S87-S95, 2010.

BOTEGA, Neury José. Comportamento suicida: epidemiologia. Psicologia Usp, v. 25, n. 3, p. 231-236, 2014.

DA SILVA SOARES FILHO, Erivaldo et al. O suicídio no Estado do Tocantins. Revista Eletrônica Acervo Saúde, v. 11, n. 12, p. e712-e712, 2019.

DAUDT, Arthur Dondonis et al. Manejo em emergência do paciente suicida. Acta méd. (Porto Alegre), p. 6-6, 2014.

D'OLIVEIRA, Carlos Felipe et al. Prevenção do suicídio: manual dirigido a profissionais das equipes de saúde mental. 2006.

DURKHEIM, Émile. As Regras do Método Sociológico, (3ª edição). Lisboa: Editorial, 2007.

FEDERAL, GOVERNO DO DISTRITO; INTEGRAÇÃO, COORDENAÇÃO DE ATENÇÃO SECUNDÁRIA E. PLANO DISTRITAL DE PREVENÇÃO AO SUICÍDIO 2020-2023. 2019.

FERREIRA JUNIOR, Avimar. O comportamento suicida no Brasil e no mundo. Revista Brasileira de, 2015.

GOMES, Helierson e $t$ a $l$. PERFIL E TENDÊNCIA DOS CASOS DE SUICÍDIO NO MUNICÍPIO DE ARAGUAÍNA TOCANTINS. DESAFIOS-Revista Interdisciplinar da Universidade Federal do Tocantins, v. 7, n. 3, p. 124-133, 2020.

LIMA, Lucas Pereira et al. Aspectos epidemiológicos de mortes por suicídio no estado do Tocantins. Revista de Patologia do Tocantins, v. 6, n. 3, 2019.

LÖWY, Michael. Um Marx insólito. In MARX, Karl. Sobre o suicídio. São Paulo:

Boitempo, 2006. Cap.1, p.13-19, v. 5.

MARCOLAN, João Fernando; DA SILVA, Daniel Augusto. O comportamento suicida na realidade brasileira: aspectos epidemiológicos e da política de prevenção. Revista M. Estudos sobre a morte, os mortos e o morrer, v. 4, n. 7, p. 31-44, 2019.

MOREIRA, Roberta Magda Martins et a $l$. Análise epidemiológica dos óbitos por suicídio. SANARE-Revista de Políticas Públicas, v. 16, 2017.

ORGANIZAÇÃO MUNDIAL DA SAÚDE. CID-10: Classificação Estatística Internacional de Doenças com disquete Vol. 1. Edusp, 1994.

PERES, A. L. et al. Morte silenciada: o suicídio e a representação social. Rev. Ambiente Acadêmico, Itapemirim, v. 2, n. 1, p. 109-124, 2016.

RIBEIRO, Daniel Mendelski. Suicídio: critérios científicos e legais de análise. Jus Navigandi,v. 
9, 2004.

SADOCK, Benjamin J.; SADOCK, Virginia A.; RUIZ, Pedro. Medicina psiquiátrica de emergência. In: Compêndio de Psiquiatria: Ciência do Comportamento e Psiquiatria Clínica. 11.ed. Porto Alegre: Artmed Editora, 2016. Cap.23, p.763-790.

SOLOMON, Andrew. Suicídio. In: O demônio do meio-dia: uma anatomia da depressão. 1.ed. São Paulo: Editora Companhia das Letras, 2014. Cap.7, p.232-271.

TENG, Chei Tung; PAMPANELLI, Mariana Bonini. O Suicídio no contexto psiquiátrico. Revista Brasileira de Psicologia, v. 2, n. 1, p. 41-51, 2015.

WHO. Suicide in the world: Global Health Estimates. 2019. Disponível em: <https://apps.who.int/iris/bitstream/handle/10665/326948/WHO-MSD-MER-19.3- eng.pdf?ua=1>. Acesso em: 29 de janeiro, 2021. 